\title{
Harmine reinforces the effects of regorafenib on suppressing cell proliferation and inducing apoptosis in liver cancer cells
}

\author{
ZI-YI CHEN $^{1^{*}}$, JIE LI $^{1 *}$, SHU-DI ZHU ${ }^{1 *}$, ZHI-DI LI ${ }^{1,2^{*}}$, JIA-LIN YU $^{1}$, \\ JIE WU ${ }^{3}$, CHONG ZHANG ${ }^{1}$ and LING-HUI ZENG ${ }^{1}$ \\ ${ }^{1}$ School of Medicine, Zhejiang University City College, Hangzhou, Zhejiang 310015; \\ ${ }^{2}$ College of Pharmaceutical Sciences, Zhejiang University, Hangzhou, Zhejiang 310058; \\ ${ }^{3}$ School of Pharmaceutical and Materials Engineering and Institute for Advanced Studies, \\ Taizhou University, Taizhou, Zhejiang 318000, P.R. China
}

Received August 21, 2021; Accepted November 30, 2021

DOI: $10.3892 /$ etm.2022.11132

\begin{abstract}
The overall outcomes for patients with advanced liver cancer are far from satisfactory, and the development of more effective therapeutic strategies for liver cancer is required. Sulforhodamine blue and colony formation assays were performed to detect the proliferation of liver certain cancer cells, including HepG2 and Hep3B. Western blotting was also preformed to detect the expression of indicated proteins, including cleaved-caspase-3, cleaved-poly (ADP-ribose) polymerase, dual-specificity tyrosine phosphorylation kinase 1A (DYRK1A), PARP-1/2, GAPDH, myeloid cell leukemia-1, phosphorylated-AKT (Ser473), caspase-3, $\alpha$-tubulin and AKT. PI staining was used to detect cell death. In the present study, DYRK1A knockdown significantly enhanced the anti-liver cancer effect of regorafenib in vitro. Furthermore, DYRK1A inhibitor harmine together with regorafenib provided synergistic anti-liver cancer activity by suppressing cell proliferation. In addition, harmine significantly enhanced regorafenib-induced cell death in liver cancer cells. It has been reported that AKT signaling is activated in regorafenib-resistant cancer cells and plays a crucial role in the regulation of cellular sensitivity to regorafenib. In the present study, AKT was activated in regorafenib-treated cells, and harmine could suppress the activation of AKT and reinforce the anti-cancer effects of regorafenib via regulating AKT in liver cancer cells. These data indicated that harmine enhanced the anti-cancer effects of regorafenib on suppressing cell proliferation and
\end{abstract}

Correspondence to: Dr Chong Zhang or Professor Ling-Hui Zeng, School of Medicine, Zhejiang University City College, 51 Huzhou Street, Hangzhou, Zhejiang 310015, P.R. China

E-mail: zhangchong@zucc.edu.cn

E-mail: zenglh@zucc.edu.cn

${ }^{*}$ Contributed equally

Key words: harmine, regorafenib, liver cancer, dual-specificity tyrosine phosphorylation kinase $1 \mathrm{~A}, \mathrm{AKT}$ inducing apoptosis in liver cancer cells via regulating the activation of AKT, and harmine plus regorafenib may be a potential therapeutic regimen for treating patients with liver cancer.

\section{Introduction}

Liver cancer is the sixth common cancer and the second leading cause of death from cancer worldwide, which is frequently diagnosed at late stage and characterized by rapid progression $(1,2)$. Patients with advanced liver cancer are ineligible for surgical resection and other potentially curative treatments (3). In addition, treatment efficacy for liver cancer is poor, due to its resistance to conventional chemotherapy $(4,5)$. After decades of searching for effective therapeutic agents for liver cancer, systemic treatment with sorafenib has been established as the standard therapy for advanced liver cancer (6). Furthermore, regorafenib has been demonstrated to have a high efficacy and safety in patients who experience liver cancer progression during sorafenib treatment (7). Regorafenib, a bi-aryl urea compound, is an antiangiogenic and antitumorigenic agent approved for the treatment of patients with advanced liver cancer during sorafenib therapy; it inhibits tumor growth by targeting multiple kinases, including vascular endothelial growth factor receptors 1-3, platelet-derived growth factor receptor $\beta$, c-KIT, RET, B-RAF, fibroblast growth factor receptor 1 and serine/threonine kinase (Raf and p38 MAPK) (8). Regorafenib has been revealed to suppress cell proliferation, invasion and angiogenesis via the ERK/NF- $\kappa$ B signaling pathway (9). However, patients with advanced liver cancer develop resistance to regorafenib via the activation of the AKT signaling pathway, and the overall outcome of patients with advanced liver cancer is far from satisfactory (10). Therefore, the development of more effective therapeutic agents and strategies for liver cancer is required.

Harmine, a naturally occurring $\beta$-carboline, is isolated from a medicinal herb traditionally used in the Middle East and North Africa, known as Peganum harmala L. (11). It has a wide range of pharmacological activities, including anti-microbial, anti-fungal, anti-oxidative and anticancer activities (12). Furthermore, harmine is an ideal drug candidate for liver 
cancer therapy, as it is selectively harmful to liver cancer cells but has minimal side effects on normal liver cells (13). Harmine possesses notable anticancer properties by targeting apoptosis, autophagy, abnormal cell proliferation, angiogenesis and metastasis (14). Harmine displays pharmacological activities by suppressing substrate phosphorylation in the dual-specificity tyrosine-regulated kinase (DYRK) family, and it exhibits the highest affinity for DYRK1A (15). Furthermore, combining harmine with other agents, such as Bcl-2 inhibitors and osimertinib, has been identified as a potential approach to overcoming resistance to chemotherapy $(16,17)$. In the present study, it was examined whether harmine combined with regorafenib may be a potential therapeutic regimen for liver cancer treatment.

\section{Materials and methods}

Materials. Harmine (cat. no. HY-N0737A) was purchased from MedChemExpress and regorafenib (cat. no. A8236) was obtained by APeXBIO Technology LLC. The primary antibodies against cleaved-caspase-3 (cat. no. 9661S), cleaved-poly (ADP-ribose) polymerase (cleaved-PARP; cat. no. 9541S; 1:1,000) and DYRK1A (cat. no. 2771S; 1:1,000) were obtained from Cell Signaling Technology, Inc. The primary antibodies against PARP-1/2 (H-250) (cat. no. sc-7150; 1:500), anti-GAPDH antibody (FL-335) (cat. no. sc-25778; 1:500), myeloid cell leukemia-1 (Mcl-1) (S-19) (cat. no. sc-819; 1:500), phosphorylated (p)-AKT (1/2/3) (Ser473) (cat. no. sc-7985; 1:500) and caspase-3 (H-277) (cat. no. sc-7148; 1:500) were purchased from Santa Cruz Biotechnology, Inc. The primary antibody against $\alpha$-tubulin (rabbit polyclonal antibody; cat. no. AF0001; 1:1,000) was obtained from Beyotime Institute of Biotechnology. The primary antibody against AKT (cat. no. 610836; 1:500) was purchased from BD Biosciences. The secondary antibodies, including DyLight ${ }^{\mathrm{TM}} 8004 \mathrm{X}$ PEG-conjugated anti-rabbit-IgG (cat. no. 5151) and DyLight ${ }^{\mathrm{TM}}$ 800 4X PEG-conjugated anti-mouse-IgG (cat. no. 5470), were obtained from Cell Signaling Technology, Inc.

Cell culture. All cell lines were obtained from the Shanghai Institute of Biochemistry and Cell Biology. HepG2 cells were maintained in DMEM (cat. no. C0006; Hangzhou Keyi Shengwu Jishu Youxian Gongsi) with 10\% FBS (cat. no. P30-3302; PAN-Biotech GmbH) and Hep3B cells were cultured in MEM (cat. no. C0032, Hangzhou KEYI) with $10 \%$ FBS and $100 \mathrm{U} / \mathrm{ml}$ penicillin/streptomycin (Gibco; Thermo Fisher Scientific, Inc.). Mycoplasma testing was performed on these cell lines, which were then determined and authenticated for genotypes using short tandem repeat DNA fingerprinting and passaged for $<6$ months (18). Liver cancer cells were divided into four groups: control group treated with DMSO, harmine treated group, regorafenib treated group, harmine plus regorafenib group.

Cytotoxicity assay. The proliferation of HepG2 and Hep3B cells was measured using sulforhodamine blue (SRB) cytotoxicity assay. The cells were inoculated into a 96-well plate at a density of $8 \times 10^{3}$ cells/well. When the cell confluence reached $30 \%$, harmine, regorafenib, harmine plus regorafenib were added into the plates. After 72 h, cells were fixed with $10 \%$ trichloroacetic acid for $8 \mathrm{~h}$ at $4{ }^{\circ} \mathrm{C}$. Next, tap water was used to wash the 96-well plates. After the wells had dried up, $0.4 \%$ SRB (cat. no. 230162-5G; Sigma-Aldrich; Merck KGaA) solution was used to stain the cells $30 \mathrm{~min}$ at room temperature. Next, the unbound dye was removed using $1 \%$ acetic acid washing in the 96-well plates. Tris-based solution $(10 \mathrm{mM})$ was used to solubilize the SRB dye. Finally, cell proliferation was detected as previously described using a microplate reader at $570 \mathrm{~nm}$ (19).

Detection of cell death. Cell death was detected using PI (cat. no. ST511; Beyotime Institute of Biotechnology) followed by flow cytometry detection as previously described (20). HepG2 and Hep3B cells were inoculated at a density of $2 \times 10^{4}$ per well into a 96-well plate and incubated with harmine $(4 \mu \mathrm{M})$ and/or regorafenib $(1 \mu \mathrm{M}$ or $2 \mu \mathrm{M})$ for $48 \mathrm{~h}$ at $37^{\circ} \mathrm{C}$. The collected cells were then fixed and permeabilized with $75 \%$ precooled ethanol at $4^{\circ} \mathrm{C}$ for $2 \mathrm{~h}$. Next, $400 \mu \mathrm{l}$ PBS containing $50 \mu \mathrm{g} / \mathrm{ml}$ RNase A (cat. no. ST579; Beyotime Institute of Biotechnology) was used to treat cells at $37^{\circ} \mathrm{C}$ for $30 \mathrm{~min}$. Subsequently, cells were incubated with $5 \mu \mathrm{l}$ PI solution for $15 \mathrm{~min}$ at room temperature and analyzed using a FACSCalibur cytometer (FACSCalibur; BD Biosciences). Finally, the sub-G1 peak was analyzed by Cellquest Pro Software (version 6.0; BD Biosciences), and gating was performed to keep cell death $<10 \%$ in the control group.

Colony formation assay. HepG2 and Hep3B cells were inoculated into a $6-\mathrm{cm}$ dish at a low cell density $\left(6 \times 10^{3}\right.$ cells per dish) to evaluate the ability of cells to form colonies. Then, the cells were incubated with $1 \mu \mathrm{M}$ harmine, $2 \mu \mathrm{M}$ regorafenib and harmine plus regorafenib for 14 days at $37^{\circ} \mathrm{C}$. The sensitivity of drug treatment is dependent on cell density, drug treatment was indicated to achieve maximal anti-proliferative effect at low cell density and retain the anti-proliferative effect at intermediate cell density (data not shown). Thus, the concentrations of harmine and regorafenib used in colony formation assay were different compared with that in apoptotic detection. Dishes were fixed with $10 \%$ paraformaldehyde solution for $30 \mathrm{~min}$ at room temperature and stained with $1 \%$ crystal violet solution for $30 \mathrm{~min}$ at room temperature, and colonies containing $>50$ cells were counted using ImageJ software (National Institutes of Health; version v1.8.0). The survival fractions were calculated according to the following equation: Survival fractions $=$ cell number of treated sample/cell number of control (21).

Western blot analysis. HepG2 and Hep3B cells were incubated with harmine/regorafenib for $48 \mathrm{~h}$ at $37^{\circ} \mathrm{C}$. Cells were incubated with lysis buffer (cat. no. P0013, Beyotime Institute of Biotechnology) on ice for $30 \mathrm{~min}$. The concentrations of protein were determined by the BCA method using an Enhanced BCA Protein Assay kit (cat. no. P0009; Beyotime Institute of Biotechnology). Proteins (20 $\mu \mathrm{g} /$ lane) were fractionated on 8-12\% Tris-glycine gels, and following electrophoresis, proteins were transferred to PVDF membranes. The membranes were blocked with $5 \%$ skimmed milk for $2 \mathrm{~h}$ at room temperature and then washed three times with $0.1 \%$ TBS-Tween-20 (TBST), followed by incubation with the primary antibodies (cleaved-caspase-3, cleaved-PARP, 
A

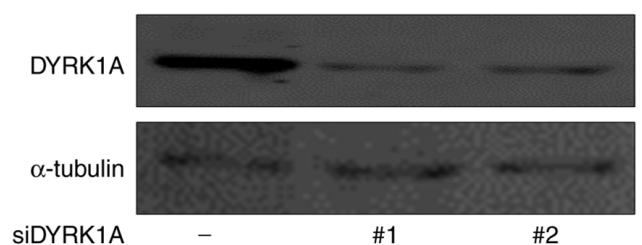

B

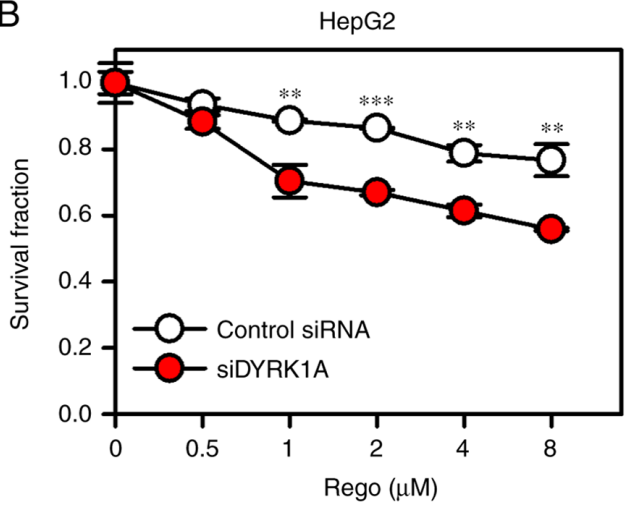

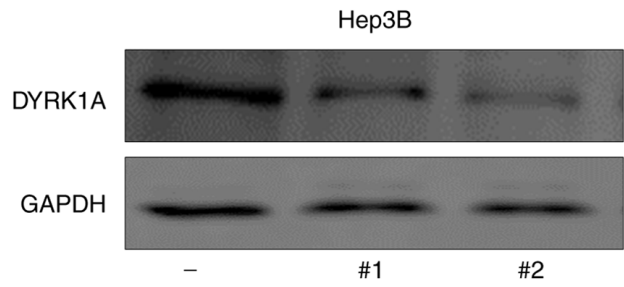

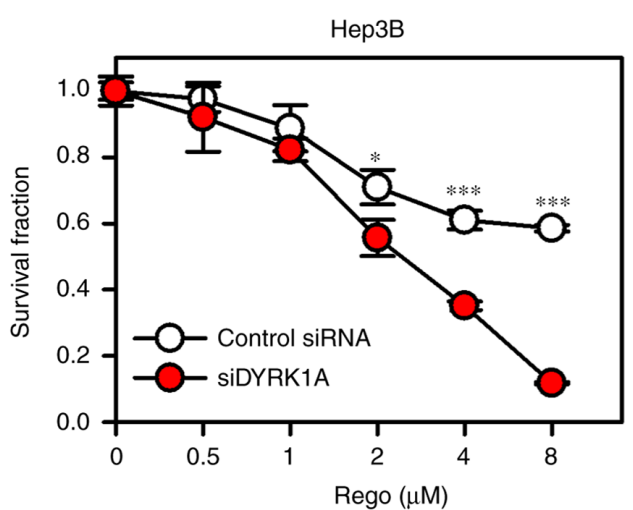

Figure 1. DYRK1A knockdown reinforces the anti-liver cancer effects of regorafenib in vitro. (A) DYRK1A was knocked down by transfecting liver cancer cells with DYRK1A siRNA-1, DYRK1A siRNA-2 or control siRNA for $48 \mathrm{~h}$, and western blot analysis was performed to detect DYRK1A expression. (B) Liver cancer cells were first transfected with DYRK1A or control siRNA for $24 \mathrm{~h}$, and then treated with regorafenib for $72 \mathrm{~h}$. Cell proliferation was determined using sulforhodamine blue assay. A two-tailed unpaired Student's t-test was used to determine the differences between control siRNA and DYRK1A siRNA groups in liver cancer cells treated with the indicated concentrations of regorafenib. ${ }^{*} \mathrm{P}<0.05,{ }^{* *} \mathrm{P}<0.01$ and ${ }^{* * * *} \mathrm{P}<0.001$ control siRNA vs. siDYRK1A. Rego, regorafenib; DYRK1A, dual-specificity tyrosine phosphorylation-regulated kinase 1A; si, small interfering.

DYRK1A, PARP-1/2, GAPDH, Mcl-1, p-AKT (Ser473), caspase-3, $\alpha$-tubulin and AKT) overnight. Next, membranes were washed three times with TBST, incubated and visualized with DyLight ${ }^{\mathrm{TM}} 800$ 4X PEG-conjugated anti-rabbit-IgG (cat. no. 5151) and DyLight ${ }^{\mathrm{TM}} 8004 \mathrm{X}$ PEG-conjugated anti-mouse-IgG (cat. no. 5257) (1:50,000; Cell Signaling Technology, Inc.) secondary antibodies for $2 \mathrm{~h}$, washed three times again with TBST and scanned using an imaging system (Odyssey CLX Image Studio; version 5.0.21; LiCor Odyssey CLx imager; LI-COR Biosciences).

RNA interference. HepG2 and Hep3B cells were seeded in six-well plates $\left(2 \times 10^{5}\right.$ cells/well $)$ and then transfected with 40 nM DYRK1A small interfering (si)RNA using jetPRIME (Polyplus-transfection SA) at $37^{\circ} \mathrm{C}$. After $24 \mathrm{~h}$ siRNA transfection, HepG2 and Hep3B cells were collected and seeded on 96 -well plates $\left(4 \times 10^{3}\right.$ cells/well) at $37^{\circ} \mathrm{C}$ overnight. Cells were then treated with regorafenib $(0.5,1,2,4$ and $8 \mu \mathrm{M})$ for $72 \mathrm{~h}$, and cell proliferation was detected using an SRB assay, as described above. For the detection of western blotting, after $24 \mathrm{~h}$ siRNA transfection, HepG2 and Hep3B cells were collected and incubated with lysis buffer, followed by western blot analysis. DYRK1A siRNAs were synthetized by Shanghai GenePharma Co., Ltd. The siRNA sequences used were as follows: siDYRK1A-1, 5'-AUGGAGCUAUGGACGUUAADT DT-3'; siDYRK1A-2, 5'-AAACUCGAAUUCAACCUUADT DT-3'; and negative control, 5'-UUCUCCGAACGUGUCACG UDTDT-3'.

Plasmid transfection. HepG2 cells reached $80 \%$ confluence in a $6-\mathrm{cm}$ plate prior to transfection. Attractene transfection reagent was purchased from Qiagen AB. Constitutively active AKT1 (CA-AKT; cat. no. 78778; pcDNA3.1-HA AKT1) plasmid was obtained from Addgene, Inc. CA-AKT or pcDNA3.1 plasmid (cat. no. V87020; Invitrogen; Thermo Fisher Scientific, Inc.) transfection was performed using Attractene transfection reagent with $1 \mu \mathrm{g}$ plasmid per reaction at $37^{\circ} \mathrm{C}$ for $24 \mathrm{~h}$, according to the manufacturer's protocol (22). Cells were then treated with $4 \mu \mathrm{M}$ harmine $+1 \mu \mathrm{M}$ regorafenib for $48 \mathrm{~h}$, after which the expression of the indicated proteins were detected.

Statistical analysis. All data are presented as the mean \pm SD. All experiments were conducted at least three times, and representative results are presented. A two-tailed unpaired Student's t-test was used to determine the differences between two groups. One-way ANOVA followed by Tukey's post hoc test was used to examine the significant differences among multiple groups using GraphPad (Version 6.01; GraphPad Software). The combination index (CI) values were calculated using CalcuSyn (Version 2.0; Biosoft; synergism, $\mathrm{CI}<0.9$; additive effect, 0.9-1.10; antagonism, >1.10). $\mathrm{P}<0.05$ was considered to indicate a statistically significant difference.

\section{Results}

DYRK1A knockdown reinforces the anticancer effects of regorafenib in vitro. DYRK1A serves a vital role in drug sensitivity in cancer treatment $(17,16)$. In the present study, two DYRK1A siRNA significantly suppressed the expression of DYRK1A and DYRK1A knockdown by siDYRK1A-1 reinforced the anticancer effects of regorafenib in liver cancer cells by suppressing cell proliferation (Fig. 1A and B). 
A
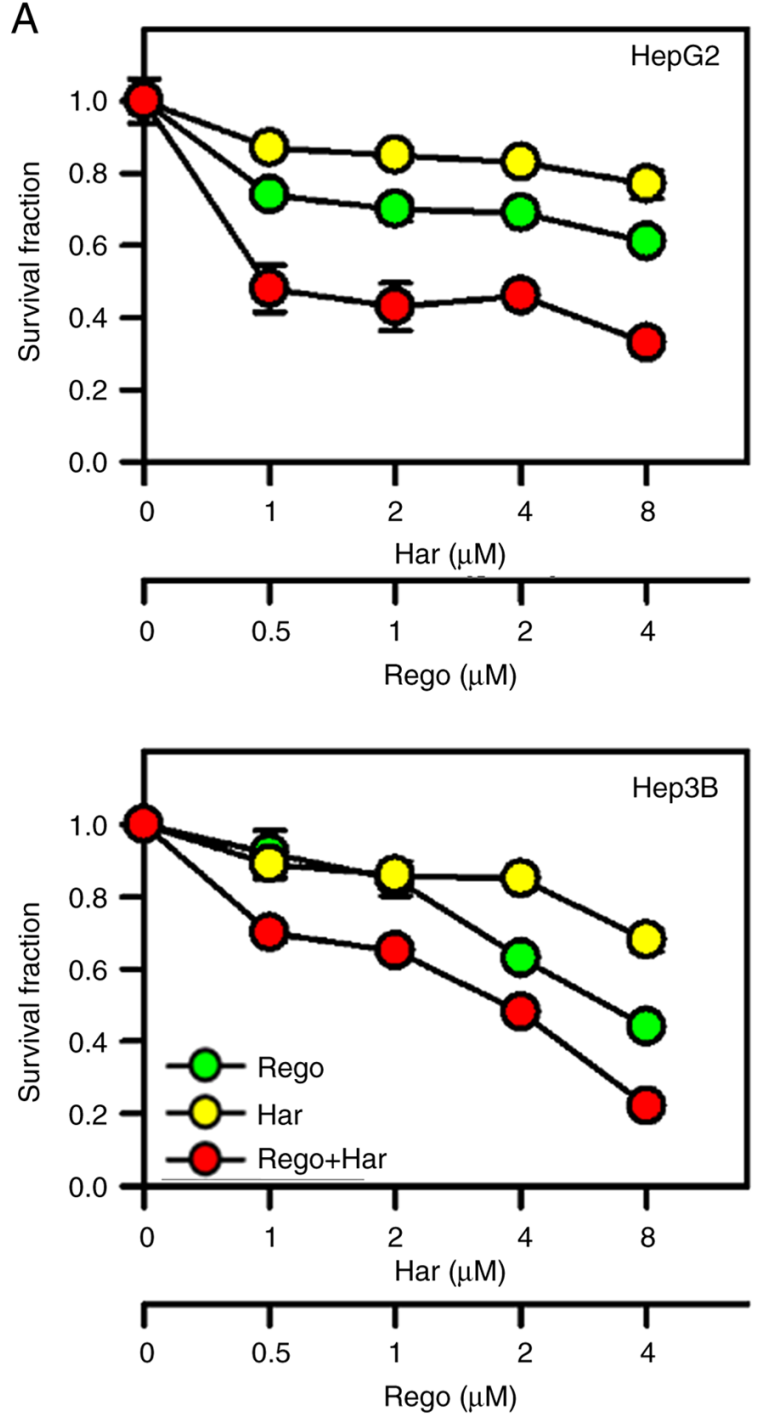

B

\begin{tabular}{ccc}
\multicolumn{4}{c}{ HepG2 } \\
\hline Rego $(\mu \mathrm{M})$ & Har $(\mu \mathrm{M})$ & $\mathrm{Cl}$ \\
\hline 0.5 & 1 & 0.016 \\
1 & 2 & 0.015 \\
2 & 4 & 0.046 \\
4 & 8 & 0.012 \\
\hline \multicolumn{3}{c}{ Mean } \\
\hline
\end{tabular}

\begin{tabular}{ccc}
\multicolumn{4}{c}{ Hep3B } \\
\hline Rego $(\mu \mathrm{M})$ & Har $(\mu \mathrm{M})$ & $\mathrm{Cl}$ \\
\hline 0.5 & 1 & 0.39 \\
1 & 2 & 0.62 \\
2 & 4 & 0.66 \\
4 & 8 & 0.50 \\
\hline & Mean & 0.5425 \\
\hline
\end{tabular}

Figure 2. Harmine plus regorafenib synergistically inhibit the proliferation of liver cancer cells. (A) Liver cancer cells were incubated with harmine and/or regorafenib for $72 \mathrm{~h}$, and cell proliferation was then determined using sulforhodamine blue assay. (B) The mean CI values were calculated and are demonstrated. Rego, regorafenib; Har, harmine; CI, combination index.

Harmine plus regorafenib synergistically inhibits the proliferation of liver cancer cells. As expected, the DYRK1A inhibitor harmine plus regorafenib significantly suppressed the proliferation of liver cancer cells compared with single-agent treatment (Fig. 2A). To verify the synergistic anti-liver cancer effect of harmine plus regorafenib, CI values were calculated. As revealed in Fig. 2B, co-treatment with harmine and regorafenib exhibited synergistic anti-proliferative effects on liver cancer cells $(\mathrm{CI}<0.7)$. Particularly in $\mathrm{HepG} 2$ cells, harmine plus regorafenib exhibited a strong synergy $(\mathrm{CI}<0.1)$. In addition, harmine could increase the suppression of colony formation by regorafenib in liver cancer cells (Fig. 3). Thus, these data revealed that harmine enhanced the anticancer effects of regorafenib by inhibiting liver cancer cell proliferation.

Harmine increases regorafenib-induced cell death. To further confirm the synergistic anti-liver cancer effect of harmine plus regorafenib, PI staining was used to detect cell death. As demonstrated in Fig. 4A, the cell death proportion of HepG2 cells (sub-G1) was $3.20 \%$ in the control group, $9.98 \%$ in the regorafenib group, $49.81 \%$ in the harmine group and $80.40 \%$ in the combination treatment group. Therefore, harmine markedly enhanced regorafenib-induced cell death in HepG2 cells (Fig. 4B). The enhanced cell death induced by harmine plus regorafenib was also observed in Hep3B cells, compared with single agent treatment. These data revealed that harmine plus regorafenib significantly induced cell death in liver cancer cells.

Harmine combined with regorafenib induces cell death via the AKT pathway. Furthermore, western blot analysis detecting cleaved-PARP and cleaved-caspase- 3 confirmed that harmine plus regorafenib treatment promoted apoptosis in liver cancer cells when compared with single agent treatment (Fig. 5A and B). The phosphorylation of AKT was enhanced in liver cancer cells treated with regorafenib compared with the DMSO treated group, while harmine plus regorafenib significantly suppressed p-AKT compared with single agent treatment in HepG2 and Hep3B cells. Furthermore, harmine plus regorafenib significantly inhibited the expression of Mcl-1 compared with single agent treatment. 

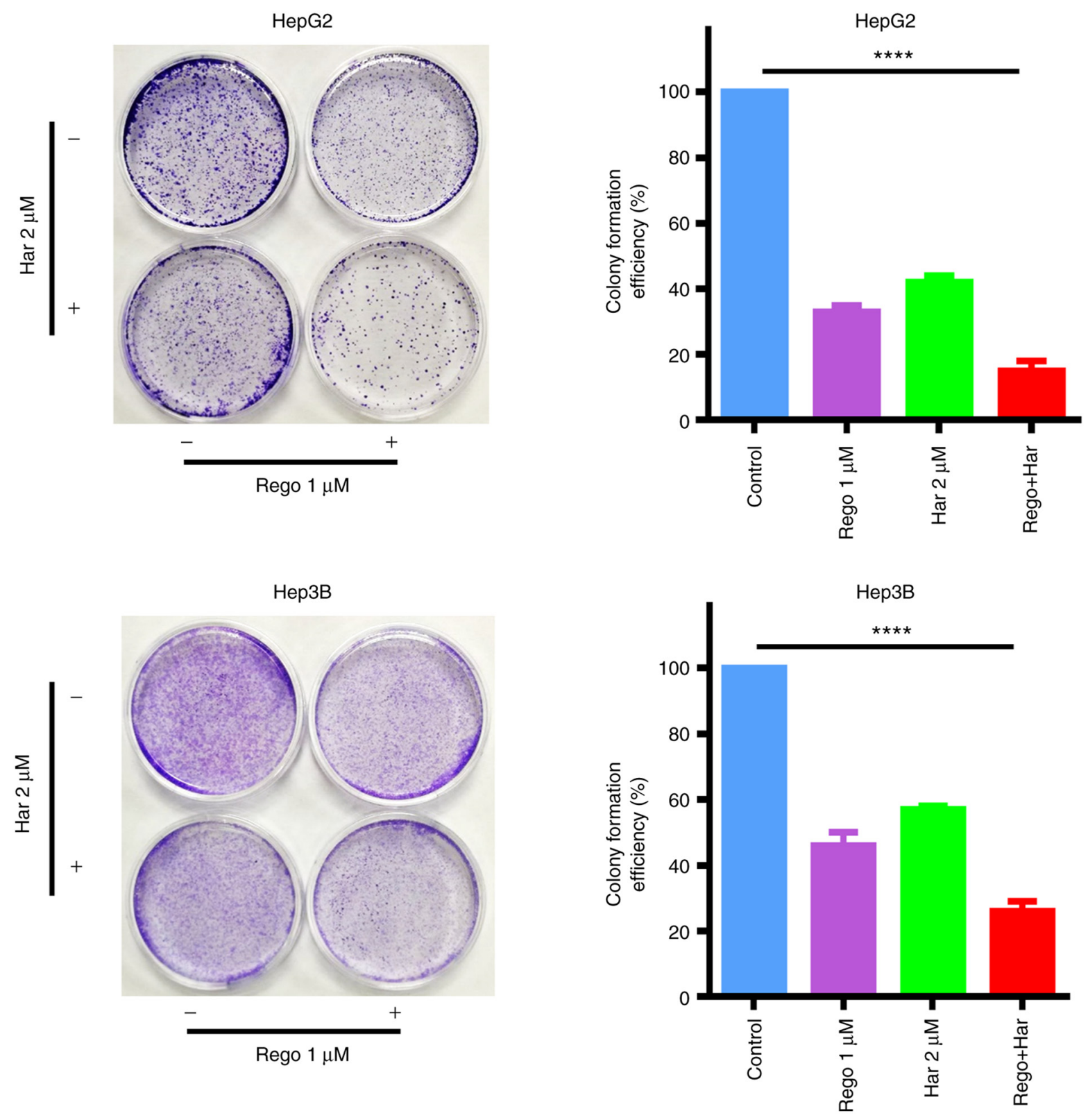

Figure 3. Harmine increases the anti-cancer proliferative effects of regorafenib. Liver cancer cells were incubated with harmine/regorafenib for 14 days and underwent crystal violet staining. One-way ANOVA followed by Tukey's post hoc test was used to examine the significant differences among four groups, including control, regorafenib, harmine and regorafenib plus harmine groups. ${ }^{* * * *} \mathrm{P}<0.0001$. Rego, regorafenib; Har, harmine.

Overexpression of AKT reverses the apoptosis induced by regorafenib plus harmine in liver cancer cells. To determine the role of the AKT signaling pathway in regorafenib plus harmine treatment, AKT was overexpressed by transfecting the CA-AKT plasmid into liver cancer cells. Regorafenib plus harmine induced apoptosis in the AKT-overexpression group was lower than that in empty vector group, indicating that AKT overexpression reduced the apoptosis induced by regorafenib plus harmine in liver cancer cells (Fig. 6A and B). Thus, these results demonstrated that AKT signaling may serve a vital role in regorafenib plus harmine treatment.

\section{Discussion}

The activation of the $\mathrm{PI} 3 \mathrm{~K} / \mathrm{AKT} / \mathrm{mTOR}$ pathway is involved in the development and proliferation of liver cancer stem cells during acquired sorafenib resistance (23). Although regorafenib is the only systemic therapy demonstrated to provide survival advantages in patients with liver cancer experiencing disease progression on sorafenib treatment, the activation of the AKT pathway still restricts the anticancer activity of regorafenib during acquired regorafenib resistance $(24,25)$. This highlighted the need for alternative therapeutic strategies for targeting the PI3K/AKT/mTOR pathway during sorafenib/regorafenib treatment. Mcl-1 is vital to cancer treatment due to its upregulation in a wide variety of human cancer types, including non-small-cell lung cancer, breast cancer, ovarian cancer, prostate cancer and pancreatic cancer $(26,27)$. The PI3K/AKT/mTOR pathway could increase the stabilization of the Mcl-1 protein by inhibiting Mcl-1 phosphorylation in human melanoma (28). It 

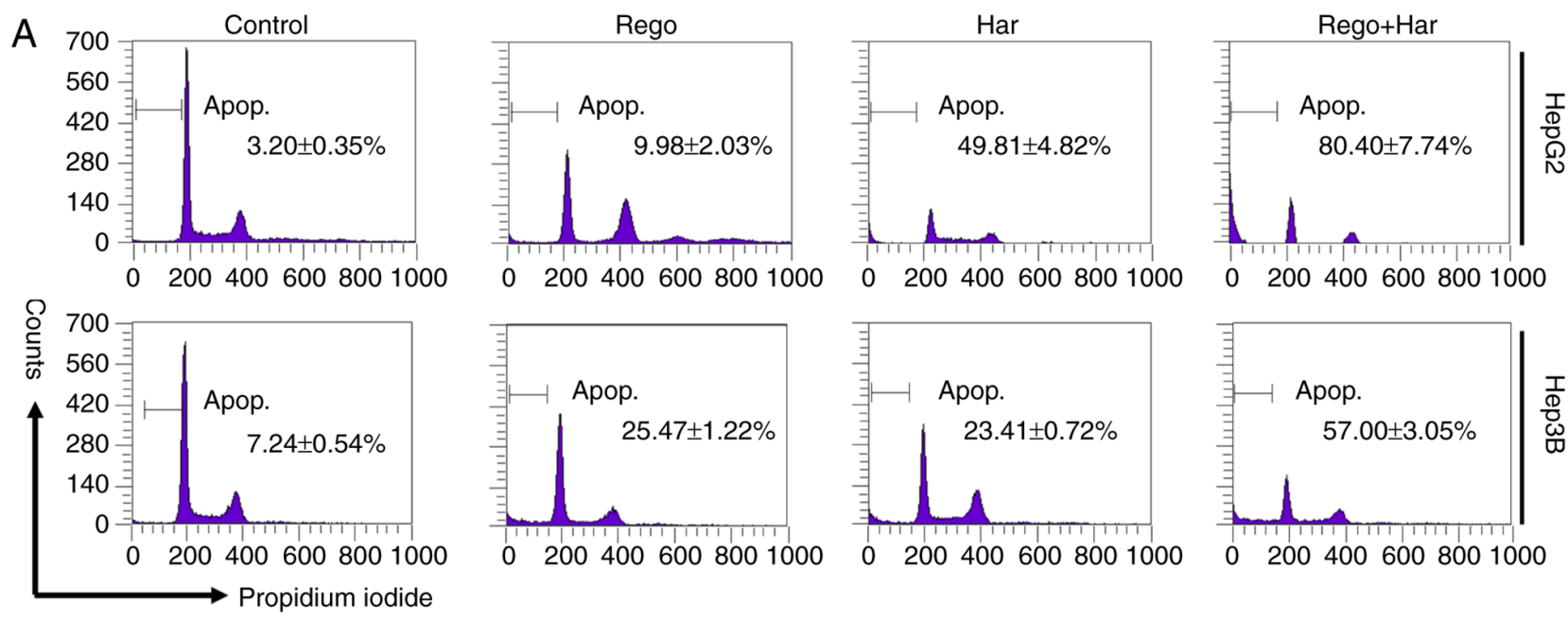

B

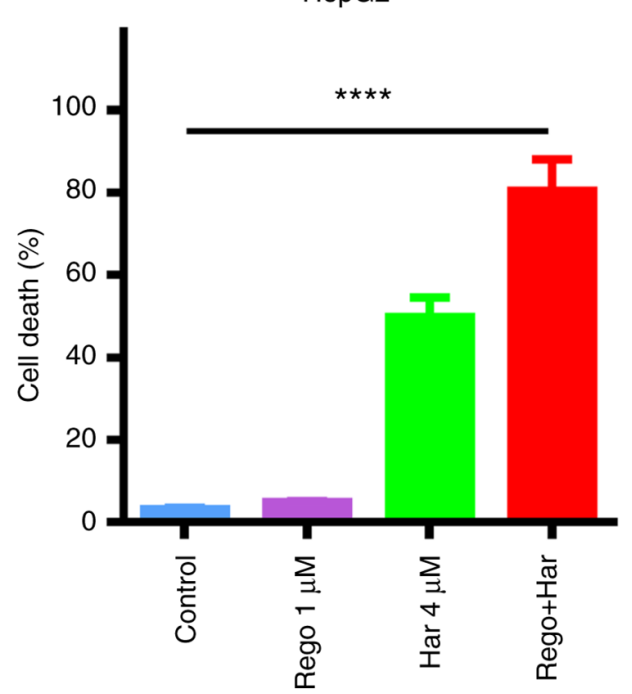

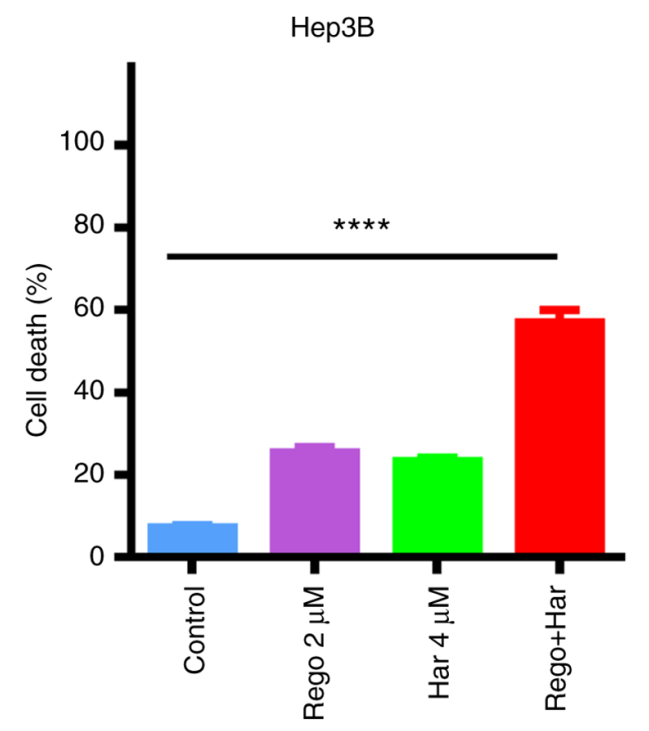

Figure 4. Harmine increases regorafenib-induced cell death. (A) Cells were incubated with harmine/regorafenib for 48 h, and propidium iodide staining was used to measure cell death. (B) Quantification of cell death as determined by flow cytometry. One-way ANOVA followed by Tukey's post hoc test was used to examine the significant differences among four groups, including control, regorafenib, harmine and regorafenib plus harmine groups. ${ }^{* * * *} \mathrm{P}<0.0001$. Rego, regorafenib; Har, harmine.

has been reported that DYRK1A overexpression has no effect on hepatic PI3K/AKT activation in mouse liver (29). However, the AKT pathway is activated by DYRK1A in the brain of mice with hyperhomocysteinemia, and treatment with harmine has been demonstrated to diminish AKT activation by reducing AKT phosphorylation (30). Furthermore, trophinin associated protein has been indicated to directly bind to DYRK1A or DYRK1B, leading to the cytoplasmic retention of DYRK1A or DYRK1B and inducing cell cycle progression via AKT activation (31). Therefore, it was suggested that AKT may be abnormally activated by DYRK1A during the pathogenesis of multiple diseases or development of drug resistance. The results of the present study indicated that AKT was activated in regorafenib-treated liver cancer cells, and that DYRK1A inhibition by harmine could suppress $\mathrm{p}-\mathrm{AKT}$ and reinforce the anti-liver cancer activity of regorafenib by inhibiting the AKT pathway. These data highlighted that harmine may be a compound that could reverse regorafenib resistance during liver cancer treatment. However, the effect of harmine plus regorafenib treatment on normal liver cells may require further investigation.

Liver cancer is closely associated with fibrosis and chronic inflammation arising from different etiologies, including alcoholic and non-alcoholic fatty liver disease and hepatitis B and C (32). Furthermore, $80-90 \%$ of patients with liver cancer have underlying cirrhosis caused by chronic liver inflammation (33). Liver cancer develops in an intricate microenvironment characterized by chronic inflammation (34). Thus, effective therapeutic approaches for liver cancer are expected to prevent chronic inflammation and oncogene-activated liver cancer growth (35). DYRK1A serves a critical role in regulating the balance between $\mathrm{T}$ helper 17 and $\mathrm{T}$ regulatory (Treg) cells, thereby contributing to the progression of inflammatory disease, and harmine attenuates inflammation by regulating Treg cell differentiation (36). Furthermore, DYRK1A suppression has been indicated to destabilize EGFR and reduce EGFR-dependent glioblastoma growth, and the pharmacological inhibition of DYRK1A has been revealed to inhibit stem cell behavior (37). These 


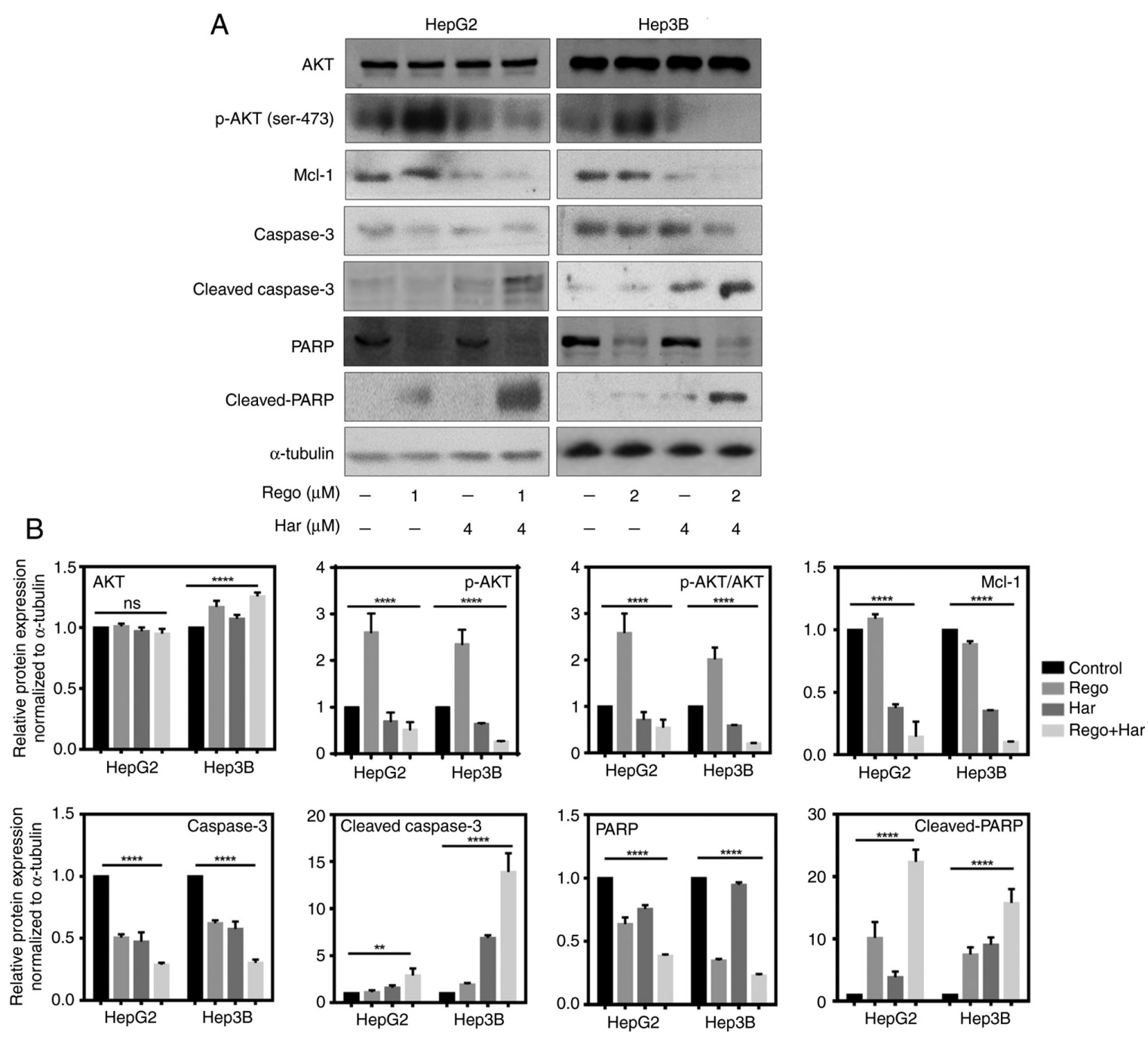

Figure 5. Harmine combined with regorafenib induces cell death via the AKT pathway. (A) Liver cancer cells were incubated with harmine/regorafenib for $48 \mathrm{~h}$ and western blot analysis was performed to detect protein expression. (B) The expression of indicated proteins was determined. One-way ANOVA followed by Tukey's post hoc test was used to examine the significant differences among four groups, including control, regorafenib, harmine and regorafenib plus harmine groups. " $\mathrm{P}<0.01$ and ${ }^{* * * * *} \mathrm{P}<0.0001$. ns, no significance; Rego, regorafenib; Har, harmine; Mcl-1, myeloid cell leukemia-1; PARP, poly (ADP-ribose) polymerase; p-, phosphorylated.
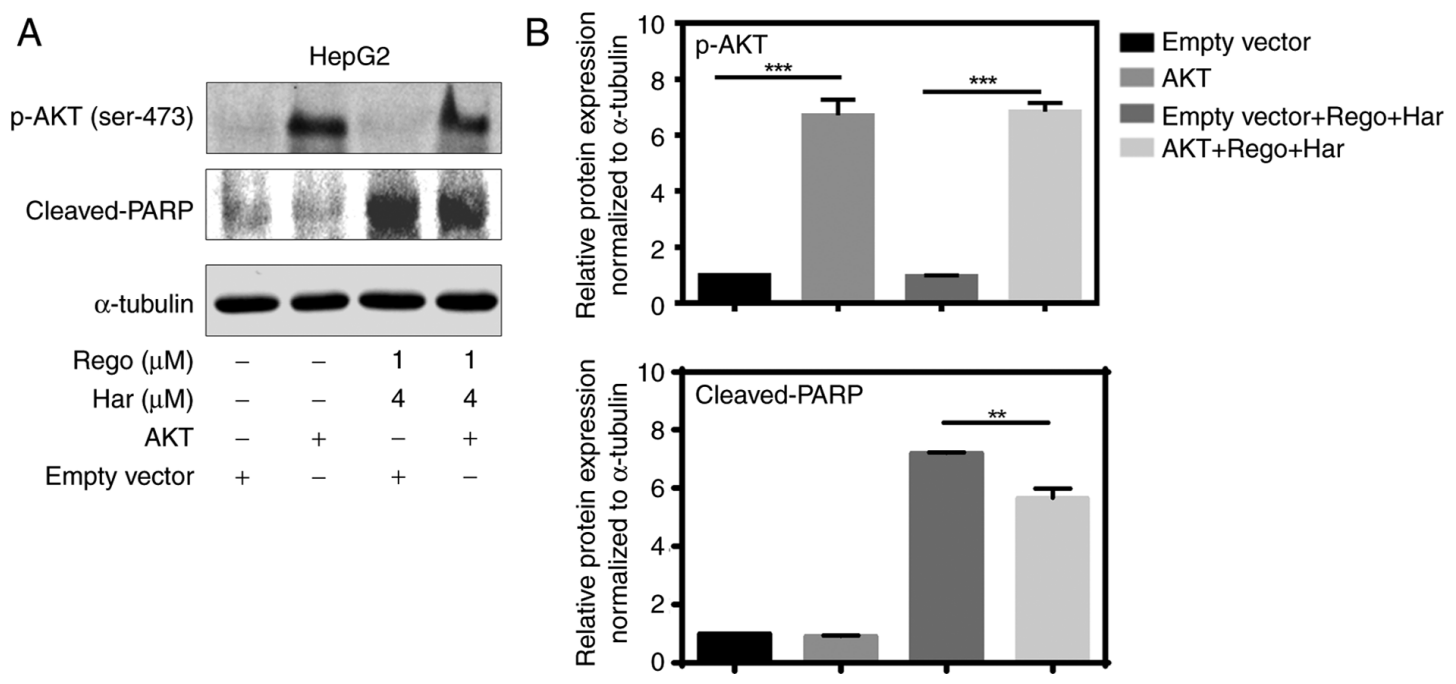

Figure 6. AKT overexpression rescues the effect of harmine plus regorafenib in liver cancer cells. (A) Transfected liver cancer cells were incubated with harmine/regorafenib for $48 \mathrm{~h}$ and western blot analysis was performed to detect protein expression. (B) The expression of the indicated proteins was determined. A two-tailed unpaired Student's t-test was used to determine the differences between two groups. ${ }^{* *} \mathrm{P}<0.01$ and ${ }^{* * *} \mathrm{P}<0.001$. p-, phosphorylated; PARP, poly (ADP-ribose) polymerase; Rego, regorafenib; Har, harmine; Rego + Har, regorafenib + harmine. 


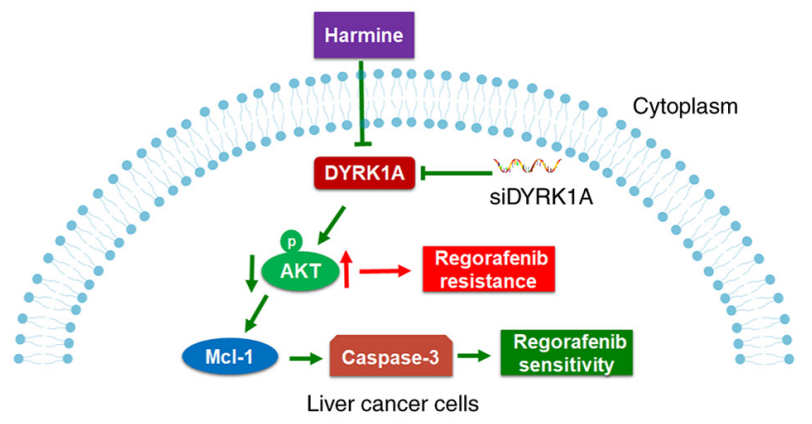

Figure 7. Effect and mechanism of harmine plus regorafenib. DYRK1A, dual-specificity tyrosine phosphorylation-regulated kinase 1A; Mcl-1, myeloid cell leukemia-1; si-, small interfering.

findings highlight the potential importance of DYRK1A in liver carcinogenesis and the need for the development of therapeutic strategies to target DYRK1A in liver cancer treatment. In the present study, to the best of our knowledge, it was reported for the first time that DYRK1A inhibition may be an efficient way of reinforcing the anticancer activity of regorafenib in liver cancer treatment. However, preclinical studies in vivo and clinical studies are required to verify the anti-liver cancer effect of harmine plus regorafenib. In addition, the efficiency of DYRK1A suppression plus regorafenib on inflammation during liver cancer development requires further investigation.

In conclusion, DYRK1A knockdown increased the anti-proliferative activity of regorafenib, and harmine enhanced the effects of regorafenib in liver cancer cells by suppressing cell proliferation and inducing apoptosis. Furthermore, harmine suppressed the expression of p-AKT and enhanced the anticancer activity of regorafenib via regulating the AKT pathway (Fig. 7). Thus, harmine may be a pertinent sensitizer to regorafenib, and harmine plus regorafenib may be an effective strategy for liver cancer treatment.

\section{Acknowledgements}

Not applicable.

\section{Funding}

The present study was supported by Zhejiang Provincial Natural Science Foundation of China (grant nos. LTY21H160001, LY21H160017 and HDMY22H160421), Public-service Technology Research Plan of Zhejiang Province (grant no. LGF21H310002), Scientific and Technological Developing Scheme of Hangzhou City (grant no. 20191203B49), Zhejiang Provincial Medical and Health Technology Project (grant nos. 2021433724 and 2020RC026) and National College Student Innovation and Entrepreneurship Training Program (grant no. 202113021025).

\section{Availability of data and materials}

All data generated or analyzed during this study are included in this published article.

\section{Authors' contributions}

CZ and LZ designed the study. ZC, ZL, JL, SZ and JY performed the experiments. JW performed the statistical analysis. CZ wrote the manuscript. ZC and ZL confirmed the authenticity of all the raw data. All authors have read and approved the final manuscript.

\section{Ethics approval and consent to participate}

Not applicable.

\section{Patient consent for publication}

Not applicable.

\section{Competing interests}

The authors declare that they have no competing interests.

\section{References}

1. Calderaro J, Ziol M, Paradis V and Zucman-Rossi J: Molecular and histological correlations in liver cancer. J Hepatol 71: 616-630, 2019.

2. Gomes MA, Priolli DG, Tralhão JG and Botelho MF: Hepatocellular carcinoma: Epidemiology, biology, diagnosis, and therapies. Rev Assoc Med Bras (1992) 59: 514-524, 2013 (In English, Portuguese).

3. Wu Q and Qin SK: Features and treatment options of Chinese hepatocellular carcinoma. Chin Clin Oncol 2: 38, 2013.

4. Avila MA, Berasain C, Sangro B and Prieto J: New therapies for hepatocellular carcinoma. Oncogene 25: 3866-3884, 2006.

5. Fu J and Wang $\mathrm{H}$ : Precision diagnosis and treatment of liver cancer in China. Cancer Lett 412: 283-288, 2018.

6. Zhu YJ, Zheng B, Wang HY and Chen L: New knowledge of the mechanisms of sorafenib resistance in liver cancer. Acta Pharmacol Sin 38: 614-622, 2017.

7. Fondevila F, Méndez-Blanco C, Fernández-Palanca $P$, González-Gallego J and Mauriz JL: Anti-tumoral activity of single and combined regorafenib treatments in preclinical models of liver and gastrointestinal cancers. Exp Mol Med 51: 1-15, 2019.

8. Wilhelm SM, Dumas J, Adnane L, Lynch M, Carter CA, Schütz G, Thierauch KH and Zopf D: Regorafenib (BAY 73-4506): A new oral multikinase inhibitor of angiogenic, stromal and oncogenic receptor tyrosine kinases with potent preclinical antitumor activity. Int J Cancer 129: 245-255, 2011.

9. Chiang CH, Chung JG and Hsu FT: Regorefenib induces extrinsic/intrinsic apoptosis and inhibits MAPK/NF- $\mathrm{BB}$-modulated tumor progression in bladder cancer in vitro and in vivo. Environ Toxicol 34: 679-688, 2019.

10. Sun H, Feng F, Xie H, Li X, Jiang Q, Chai Y, Wang Z, Yang R, Li R and Hou J: Quantitative examination of the inhibitory activation of molecular targeting agents in hepatocellular carcinoma patient-derived cell invasion via a novel in vivo tumor model. Animal Model Exp Med 2: 259-268, 2019.

11. Li S, Wang A, Gu F, Wang Z, Tian C, Qian Z, Tang L and $\mathrm{Gu}$ Y: Novel harmine derivatives for tumor targeted therapy. Oncotarget 6: 8988-9001, 2015.

12. Prasad Kushwaha J, Baidya D and Patil S: Harmine-loaded galactosylated pluronic F68-gelucire 44/14 mixed micelles for liver targeting. Drug Dev Ind Pharm 45: 1361-1368, 2019.

13. Zhang L, Zhang F, Zhang W, Chen L, Gao N, Men Y, Xu X and Jiang Y: Harmine suppresses homologous recombination repair and inhibits proliferation of hepatoma cells. Cancer Biol Ther 16: 1585-1592, 2015

14. Jalali A, Dabaghian F and Zarshenas MM: Alkaloids of Peganum harmala: Anticancer biomarkers with promising outcomes. Curr Pharm Des 27: 185-196, 2021.

15. Seifert A, Allan LA and Clarke PR: DYRK1A phosphorylates caspase 9 at an inhibitory site and is potently inhibited in human cells by harmine. FEBS J 275: 6268-6280, 2008. 
16. Li Y, Zhou D, Xu S, Rao M, Zhang Z, Wu L, Zhang C and Lin N DYRK1A suppression restrains Mcl-1 expression and sensitizes NSCLC cells to Bcl-2 inhibitors. Cancer Biol Med 17: 387-400, 2020

17. Li YL, Ding $\mathrm{K}, \mathrm{Hu} \mathrm{X}, \mathrm{Wu}$ LW, Zhou DM, Rao MJ, Lin NM and Zhang C: DYRK1A inhibition suppresses STAT3/EGFR/Met signalling and sensitizes EGFR wild-type NSCLC cells to AZD9291. J Cell Mol Med 23: 7427-7437, 2019.

18. Li YL, Zhang NY, Hu X, Chen JL, Rao MJ, Wu LW, Li QY, Zhang B, Yan W and Zhang C: Evodiamine induces apoptosis and promotes hepatocellular carcinoma cell death induced by vorinostat via downregulating HIF-1 $\alpha$ under hypoxia. Biochem Biophys Res Commun 498: 481-486, 2018.

19. Vichai V and Kirtikara K: Sulforhodamine B colorimetric assay for cytotoxicity screening. Nat Protoc 1: 1112-1116, 2006.

20. Riccardi C and Nicoletti I: Analysis of apoptosis by propidium iodide staining and flow cytometry. Nat Protoc 1: 1458-1461, 2006.

21. Zhang C, Shi J, Mao SY, Xu YS, Zhang D, Feng LY, Zhang B, Yan YY, Wang SC, Pan JP, et al: Role of p38 MAPK in enhanced human cancer cells killing by the combination of aspirin and ABT-737. J Cell Mol Med 19: 408-417, 2015.

22. Zhang D, Yan B, Yu S, Zhang C, Wang B, Wang Y, Wang J, Yuan Z, Zhang L and Pan J: Coenzyme Q10 inhibits the aging of mesenchymal stem cells induced by D-galactose through Akt/mTOR signaling. Oxid Med Cell Longev 2015: 867293, 2015.

23. Kahraman DC, Kahraman T and Cetin-Atalay R: Targeting PI3K/Akt/mTOR pathway identifies differential expression and functional role of IL8 in liver cancer stem cell enrichment. Mol Cancer Ther 18: 2146-2157, 2019.

24. Chen W, Yang J, Zhang Y, Cai H, Chen X and Sun D: Regorafenib reverses HGF-induced sorafenib resistance by inhibiting epithelial-mesenchymal transition in hepatocellular carcinoma. FEBS Open Bio 9: 335-347, 2019.

25. Mirone G, Perna S, Shukla A and Marfe G: Involvement of notch-1 in resistance to regorafenib in colon cancer cells. J Cell Physiol 231: 1097-1105, 2016.

26. Xiang W, Yang CY and Bai L: MCL-1 inhibition in cancer treatment. Onco Targets Ther 11: 7301-7314, 2018.

27. Wei SH, Dong K, Lin F, Wang X, Li B, Shen JJ, Zhang Q, Wang R and Zhang HZ: Inducing apoptosis and enhancing chemosensitivity to gemcitabine via RNA interference targeting Mcl-1 gene in pancreatic carcinoma cell. Cancer Chemother Pharmacol 62 1055-1064, 2008.
28. Jin L, Hu WL, Jiang CC, Wang JX, Han CC, Chu P, Zhang LJ, Thorne RF, Wilmott J, Scolyer RA, et al: MicroRNA-149*, a p53-responsive microRNA, functions as an oncogenic regulator in human melanoma. Proc Natl Acad Sci USA 108: 15840-15845, 2011.

29. Noll C, Tlili A, Ripoll C, Mallet L, Paul JL, Delabar JM and Janel N: Dyrk 1a activates antioxidant NQO1 expression through an ERK1/2-Nrf2 dependent mechanism. Mol Genet Metab 105: 484-488, 2012

30. Abekhoukh S, Planque C, Ripoll C, Urbaniak P, Paul JL, Delabar JM and Janel N: Dyrk1A, a serine/threonine kinase, is involved in ERK and Akt activation in the brain of hyperhomocysteinemic mice. Mol Neurobiol 47: 105-116, 2013.

31. Li L, Wei JR, Song Y, Fang S, Du Y, Li Z, Zeng TT, Zhu YH, Li Y and Guan XY: TROAP switches DYRK1 activity to drive hepatocellular carcinoma progression. Cell Death Dis 12: 125, 2021.

32. Yang YM, Kim SY and Seki E: Inflammation and liver cancer: Molecular mechanisms and therapeutic targets. Semin Liver Dis 39: 26-42, 2019

33. Ringelhan M, Pfister D, O'Connor T, Pikarsky E and Heikenwalder M: The immunology of hepatocellular carcinoma. Nat Immunol 19: 222-232, 2018.

34. He M, Zhang W, Dong Y, Wang L, Fang T, Tang W, Lv B, Chen G, Yang B, Huang P and Xia J: Pro-inflammation NF- $\kappa$ B signaling triggers a positive feedback via enhancing cholesterol accumulation in liver cancer cells. J Exp Clin Cancer Res 36: 15, 2017.

35. Koike K: Expression of junB is markedly stimulated by glycyrrhizin in a human hepatoma cell line. Oncol Rep 25: 609-617, 2011.

36. Khor B, Gagnon JD, Goel G, Roche MI, Conway KL, Tran K, Aldrich LN, Sundberg TB, Paterson AM, Mordecai S, et al: The kinase DYRK1A reciprocally regulates the differentiation of Th17 and regulatory T cells. Elife 4: e05920, 2015.

37. Pozo N, Zahonero C, Fernández P, Liñares JM, Ayuso A, Hagiwara M, Pérez A, Ricoy JR, Hernández-Laín A, Sepúlveda JM and Sánchez-Gómez P: Inhibition of DYRK1A destabilizes EGFR and reduces EGFR-dependent glioblastoma growth. J Clin Invest 123: 2475-2487, 2013.

This work is licensed under a Creative Commons Attribution-NonCommercial-NoDerivatives 4.0 International (CC BY-NC-ND 4.0) License. 